\title{
L'enseignement d'une langue vivante seconde à des élèves créolophones : le cas de l'anglais à La Réunion
}

\author{
Sophie Dufossé Sournin \\ Collège Trois Mares \\ Université de La Réunion
}

\section{Introduction}

L'apprentissage d'une langue seconde à La Réunion se fait dans un contexte différent de celui de la métropole française. Les élèves parlent créole à la maison et ont beaucoup de difficultés (pour nombre d'entre eux) à s'adresser dans un français correct aux adultes de l'Éducation nationale. Martinez explique ce problème de diglossie par une opposition «vernaculaire / véhiculaire». Pour lui,

«Le vernaculaire est une langue ou une variété telle qu'un dialecte, utilisée au foyer familial, à la maison, tandis que le véhiculaire dépasse le cadre de vie d'une communauté linguistique et répond à un besoin social d'intercommunication entre groupes dotés de vernaculaires spécifiques. L'espagnol, l'anglais, le français, bien sûr, jouent ce rôle, tout comme les créoles dans la Caraỉbe ou l'océan Indien ou le lingala en Afrique » (Martinez 19).

Les performances aux évaluations nationales le prouvent, leur langue maternelle est un réel obstacle à l'acquisition du français. Les recherches effectuées ces vingt dernières années font penser que les acteurs de la politique éducative locale estiment que le problème de diglossie se règlera de lui-même.

Quand on sait que 95\% du «matériau lexical» créole est d'origine française, on comprend aisément les difficultés des élèves réunionnais "tiraillés » entre le parler familial et la qualité de langue exigée par l'école, surtout si l'on considère, d'après $\mathrm{R}$. Chaudenson, que le créole serait «l'autonomisation d'approximations du français » (Beniamino 7). Les problèmes d'interférences (mauvaise compréhension du fonctionnement de la langue 2 [le français] par rapport à la langue 1 [le créole réunionnais], ainsi que les erreurs dites de "calques» L1/L2 sont excessivement courantes dans les productions orales mais aussi écrites des jeunes apprenants. Les collègues de français corrigent constamment des erreurs de type participe passé mis à la place de l'infinitif, par exemple, la notion de terminaison en «-er» du verbe du premier groupe n'existant pas en créole. Tous les sons /e/ sont écrits «-é ».

Qu'en est-il, dans ces conditions particulières, de l'acquisition de l'anglais langue seconde? Le parler créole devient-il un problème pédagogique non négligeable qui modifierait l'apprentissage linguistique au collège, au point de contrarier les résultats des recherches menées par les spécialistes?

Dans cet article, je vais tout d'abord expliquer les difficultés linguistiques spécifiques rencontrées par les enfants à La Réunion. Je vais ensuite décrire dans les détails une expérimentation menée auprès d'élèves de 11 ans, inscrits en Sixième dans un collège au sud de l'île. Elle concerne l'enseignement de l'anglais LV2 chez des pseudo-débutants. L'analyse des résultats permettra l'établissement d'un bilan à petite échelle. Je proposerai alors une réflexion quant au rôle joué par le créole réunionnais dans l'apprentissage de l'anglais en milieu scolaire.

\section{Problématique}


Il semblait assez logique de penser que l'application des dernières méthodologies linguistiques serait plus efficace en termes de rapidité d'acquisition et de fixation des phénomènes linguistiques en anglais, par exemple. On sait maintenant que le développement intellectuel des enfants ne se fait pas par stades successifs, comme l'avait démontré J. Piaget en son temps, mais plutôt par vagues inégales, avec des passages régressifs. On sait aussi, depuis les psychologues d'Europe de l'Est et, en particulier L. Vygotski (Grangeat in Ruano-Borbalan 106), que la croissance de l'enfant est ancrée dans son entourage social, et que l'apprentissage de la parole en dépend. Chaque élève d'une même classe d'âge va évoluer à son rythme mais dans le cadre précis du groupe scolaire constitué selon l'année de naissance des individus, ce qui, selon les spécialistes (pédopsychiatres, psychologues, médecins généralistes, sociologues, pédagogues, etc.) n'est pas le plus approprié. Les évolutionnistes « ont une conception dynamique de l'intelligence, qui évolue selon eux de façon graduelle et chaotique » (Fournier et Lécuyer 28). Ce qui fait dire à Bailly dans le domaine de la didactique de l'anglais langue seconde, $[. .$.$] le parcours d'apprentissage de l'élève$ comportera la découverte, la saisie et l'assimilation graduelle de l'objet (langue-culture) qui lui est présenté en éclats parcellaires, et qu'on lui demande de faire sien. » (10)

Il est alors difficile de pouvoir envisager la séquence didactique en langue seconde et plus précisément en anglais, comme un temps unique et égal pour tous les élèves, pendant lequel chacun puisera et retiendra le maximum d'informations transmises. Comme l'a expliqué M. Altet, «[...] Il n'existe pas une méthode d'enseignement ou de formation bonne en soi, définissable scientifiquement, qui permettrait la réussite de tous, en tout temps et en tout lieu.

C'est plutôt la mise en oeuvre d'une méthode par le formateur dans un contexte donné qui en fait son efficacité. Il n'est pas possible de comparer ou d'évaluer les méthodes d'enseignement ou de formation pour elles-mêmes en les isolant des autres variables de la situation. » (Ruano-Borbalan 201)

D. Bailly a démontré par l'analyse rigoureuse de son enseignement de l'anglais à son élève adulte Paul, que l'apprentissage linguistique d'une langue seconde suit « une courbe en $U$ » avec des progrès, des déceptions :

« [...] ces phases, on le sait, décrivent « une courbe en $U$ », où, dans un premier stade, l'apprenant commet un grand nombre d'erreurs parce qu'il stocke des unités "itémiquement ", séparément, sans système et qu'il ne maitrise pas leur utilisation; puis les erreurs diminuent parce que l'apprenant acquiert un semblant de système, a recours à de prudentes stratégies d'évitement, et que l'apprentissage rote et rule vont cumuler positivement leurs effets; ensuite encore l'apprenant aura l'impression qu'il a dominé le système et va se livrer à tort et à travers à une multitude d'essais et d'erreurs expérimentaux, hardis et risqués: d'où la remontée du nombre d'erreurs (branche droite du U). Ce n'est qu'ultérieurement que l'apprenant pourra accéder à une maîtrise acceptable du système. » (59)

La situation linguistique à La Réunion est caractérisée par une histoire où deux langues, le créole et le français, ont entretenu de très étroites et difficiles relations. Les recherches effectuées ces vingt dernières années font penser que les acteurs de la politique éducative locale estiment que le problème de diglossie se règlera de lui-même. D'après $\mathrm{M}$. Beniamino « La situation linguistique à La Réunion est en effet aussi caractérisée par une histoire linguistique où deux langues, le créole et le français, dans sa variété régionale, ont entretenu de très étroites relations ${ }^{1} \gg(1)$. Les analyses de R. Chaudenson ont montré que «La période de la diglossie coloniale [...] qui est marquée par la fin des immigrations massives - rappelons qu'en 1867 le tiers des 210000 habitants de l'île étaient des immigrants récents -, voit la disparition du rôle médiateur du créole et le passage de cette langue à une situation de minorisation. » (Beniamino 3)

\footnotetext{
${ }^{1}$ D'après R. Chaudenson « [...] le français canadien et le parler réunionnais sont plus directement que le français populaire, les continuateurs du français parlé au XVIIe siècle, non pas la langue des personnages de Racine, mais celle de l'homme du commun. » (Beniamino 1)
} 
R. Chaudenson illustre la situation par le schéma suivant (Beniamino 3) :

Figure 1 : Autonomisation du créole réunionnais selon Chaudenson.

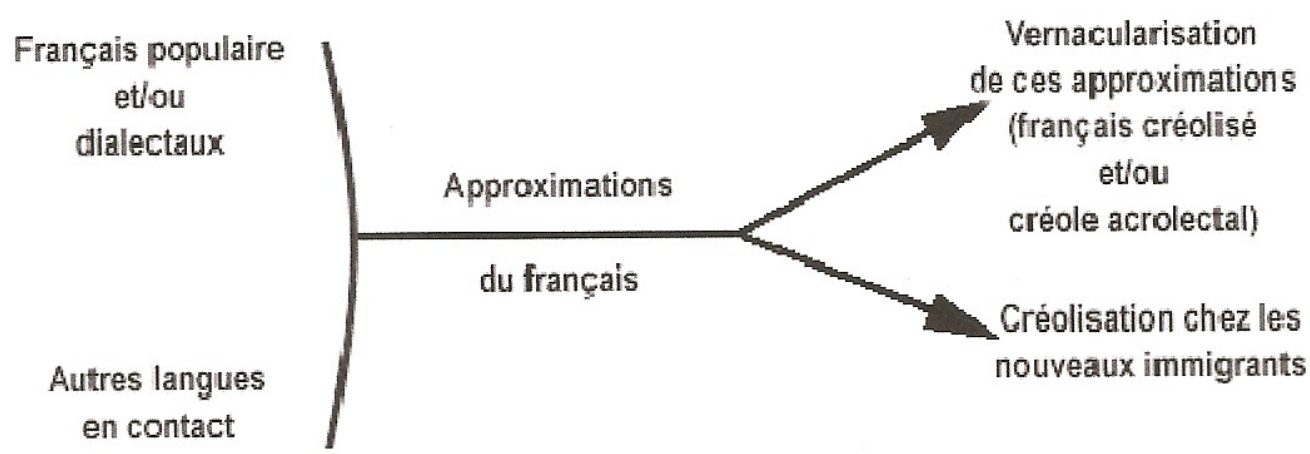

La proximité linguistique langue de région/créole (certains parlent de "français réunionnais ») implique des problèmes de calques et d'interférence néfastes à l'apprentissage du français standard de la Métropole. Les multiples difficultés liées à la situation de contacts des langues que connait La Réunion fait que les enfants ne savent souvent pas déterminer le moment où cesse le français et où commence le créole. D'après M. Beniamino,

«L'origine d'une telle perplexité réside bien sûr dans la parenté génétique du créole et du français qui produit une proximité typologique en synchronie: c'est ce que R. Chaudenson appelle une situation de «symbiose linguistique ». La genèse du créole réunionnais s'est en grande partie effectuée à partir des français populaires et/ou dialectaux parlés par les premiers colons [...] et la situation réunionnaise est donc marquée par la coexistence de deux systèmes linguistiques: le français dans ses variétés standards et non standard et un créole exogène (c'est-à-dire constitué dans une île primitivement déserte), un créole qui typologiquement est à ranger dans ce que les créolistes appellent les « créoles à base lexicale française. » (5)

\section{Cadre conceptuel}

Étant en poste dans un collège du sud de La Réunion, je me suis proposée de suivre l'évolution de six classes de Sixième. L'expérimentation a duré un semestre de l'année scolaire 2005. Elle a nécessité la mobilisation de trois enseignantes. La scolarité était répartie comme suit:

Figure 2 : Horaire hebdomadaire d'une classe de Sixième à La Réunion par discipline scolaire en 2004-2005.

\begin{tabular}{|l|c|}
\hline \multicolumn{1}{|c|}{ Disciplines scolaires } & Horaire hebdomadaire \\
\hline Français & 5 heures \\
\hline Mathématiques & 4 heures \\
\hline Histoire / géographie / instruction civique & 3 heures \\
\hline Anglais LV1 & 4 heures \\
\hline Sciences et Vie de la Terre & 2 heures \\
\hline Technologie & 1,5 heure \\
\hline Éducation physique et sportive & 4 heures \\
\hline Éducation artistique & 1 heure \\
\hline Éducation musicale & 1 heure \\
\hline
\end{tabular}


Les enfants de la classe dite bi-langue avaient - et ont toujours - trois heures d'anglais ainsi que trois heures d'une autre langue vivante, allemand ou espagnol, par semaine. Ils sont proposés pour cette option par les maittres de primaire (CM2) avec l'accord des parents. Les groupes sont composés en fin d'année, en juin, par les professeurs en charge des disciplines linguistiques de façon à ce que tout soit près à la reprise des cours. Ces heures supplémentaires de LV2 représentent une charge de travail non négligeable pour ces petits, et seuls les plus performants peuvent y avoir accès. Leur emploi du temps ne change guère en Cinquième. Ceux qui peinent à suivre cette scolarité peuvent toujours arrêter l'option en fin de Sixième sur avis du conseil de classe et du professeur principal. Ils retrouvent l'apprentissage d'une seconde LV2 en Quatrième. À La Réunion, l'année scolaire est répartie différemment de celle de la Métropole. Elle suit le calendrier « cyclonique ». La rentrée a lieu après le 15 août et le cycle se termine début juillet. Il y a des "grandes vacances» en janvier (six semaines [période cyclonique]) et en juillet-août (six semaines). Il y a des « petites vacances » en octobre, mars et mai.

Ce travail a été la mis en place en décembre 2004, lors du conseil d'enseignement de l'équipe des enseignants d'anglais, à la demande du chef d'établissement. Ils devaient trouver le moyen de suivre les apprenants débutants, tout au long du deuxième semestre de l'année scolaire en cours, afin de faire "remonter» un profil d'élève avec ses réussites et ses échecs, et modifier le projet d'établissement en conséquence. En effet, face aux résultats plutôt moyens, voire décevants, des élèves en anglais dans un département d'Outre-mer à l'époque de la mise en place, en France, du socle commun des connaissances et de l'évaluation de différentes disciplines scolaires par paliers de compétences, il était vraisemblable que certains points linguistiques se devaient d'être exploités sous un angle didactique différent.

J'ai proposé, dans le cadre de mes recherches d'utiliser un objectif grammatical : l'acquisition des articles anglais par des apprenants quasi-débutants. Le but était de maitriser l'article devant les noms dénombrables et indénombrables en repérant et identifiant des éléments grammaticaux, soit :

$-a / a n+$ dénombrables au singulier;

$-\varnothing+$ dénombrables et indénombrables;

-the + dénombrables et indénombrables.

Il s'agissait, dans ces conditions, de mettre à jour les compétences d'un élève ou d'un groupeclasse afin d'élaborer des outils pédagogiques ponctuels pour l'année scolaire et pour les suivantes. Les conclusions d'un tel travail pouvaient s'avérer fort utiles dans le cadre d'un suivi individualisé pour répondre aux besoins de chacun des élèves grâce au repérage de leurs points faibles, en adaptant la pédagogie aux résultats obtenus, voire en modifiant le projet d'établissement. Je tiens à préciser qu'en Sixième, les opérations au niveau des mots sont les suivantes :

- orthographe/morphologie/formation des mots;

- détermination nominale (déterminants de base [articles, adjectifs démonstratifs, adjectif numéral cardinal, génitif déterminatif], substituts du nom [pronoms personnels]);

- quantification (dénombrabilité, nombre, quantificateurs);

- combinaison (complémentation du nom, de l'adjectif, du verbe, expansion adverbiale éventuelle). (Bailly 165)

\section{Méthodologie}

Le déroulement du projet s'est organisé comme suit:

-évaluation diagnostique: exercice identique (lacunaire ${ }^{2}$ ou «exercice à trous») inclus dans le

${ }^{2}$ Le fait de garder le même type d'exercice et la même présentation tout au long de l'expérimentation permet de disposer d'un cadre rassurant pour les élèves et de garantir la rigueur du travail et, d'une certaine manière, des résultats. 
contrôle des élèves de plusieurs classes puis résultats chiffrés ;

-ré-explication du point de grammaire au besoin, selon les performances ;

-deuxième exercice portant sur le même point de grammaire quelques jours ou semaines après dans les évaluations des classes ciblées puis,

-résultats chiffrés

- comparaison des résultats

-adaptation de la pédagogie (reprise du point de grammaire non-acquis) ;

-troisième exercice avec rappel grammatical ;

-bilan de fin d'année : comparaison des données chiffrées des classes concernées en fonction d'un niveau requis exigible pour tous ;

-élaboration d'une stratégie didactique adaptée définitive pour l'année suivante (à titre indicatif pour les collègues).

Le choix didactique d'un exercice lacunaire vient du fait que les élèves sont habitués, depuis leur scolarité à l'école primaire, à ce type d'évaluation rapide et relativement efficace. Compléter une courte phrase par un mot ou un « outil » («a»/ «an», «the» ou $\varnothing)$ minimise à priori les risques d'erreurs, que ce soit pendant l'évaluation ou la correction. Le problème à résoudre est ainsi ciblé et évident. Chaque correction d'exercice doit inclure le rappel grammatical de l'utilisation des articles anglais étudiés en contextes dans le cadre d'une réactivation spiralaire basée sur des rebrassages $^{3}$ et des réactivations ${ }^{4}$ efficace et programmée des savoirs, soit une "progression intégrative de l'apprentissage liée à une conception constructiviste » (Quivy et Tardieu 252).

\section{Description de l'échantillon :}

Le travail a été réalisé sur un groupe de classes de Sixième. Il a permis d'évaluer l'accès à la connaissance et la fixation des acquis de l'enseignement de la L2, dès le départ au collège, même si certains élèves ont une certaine pratique lexicale et civilisationnelle à leur arrivée dans l'établissement. Cinq des six groupes de Sixième ciblés étudiaient l'anglais langue seconde à raison de quatre heures par semaine.

\section{Procédures}

Le premier exercice a été proposé en février 2005. Les élèves du collège utilisent alors le manuel New Wings $6^{*}$. Les huit premières leçons ont été étudiées. Le premier article, enseigné peu après la rentrée, est the, associé à un ordre avec mouvement de la main. P. Martinez le précise, « Le recours à la langue première aide sans doute l'apprenant à structurer ses deux systèmes et se révèle de nature à faire naître des hypothèses sur la L2» (22). Ce qui va déterminer les « bonnes » réponses des exercices à trous proposés, dépend autant de présupposés contextuels intériorisés que de règles de grammaire parfaitement assimilées. Il faut que les apprenants soient aptes à restituer des phrases «toutes faites », grammaticalement correctes et étudiées en classe. En bref, chacun doit retrouver les conditions de production et de reconnaissance du discours que constitue l'exercice. À cette période de l'année (les vacances de Noël), les élèves fixent assez facilement l'utilisation de l'article défini par un enchainement de type «transductif» dans lequel «the » ressemble phonologiquement à «le». Ils fixent assez facilement l'utilisation de ce déterminant dans le cas d'analogie français/anglais. L'absence d'article, par exemple, est associée au pluriel des noms. Les élèves peuvent lire dans leur workbook que "pour identifier plusieurs éléments on n'utilise pas d'article devant le groupe nominal » (Morel, Fiona et al. 19). L'ensemble vide est le symbole de

\footnotetext{
3 Rebrassage : « Réactivation régulière des acquis linguistiques permettant l'intégration mutuelle des acquis nouveaux et des acquis anciens dans un apprentissage conçu comme un processus de construction dynamique. » (Quivy et Tardieu 260)

4 Réactivations : « Réutilisation ponctuelle d'un acquis récent. » (Quivy et Tardieu 260)
} 
cette absence dans les formules grammaticales. Les apprenants associent donc l'absence de déterminant à l'apparition du « -s » du pluriel en fin de nom. Ils peinent à mémoriser que - ce que les grammaires scolaires appellent des exceptions - c'est-à-dire «children », «men » et «women », sont des mots « déjà » au pluriel, et que le verbe avec lequel ils s'accordent sera aussi au pluriel. Il est assez difficile de leur faire assimiler l'absence de détermination à l'indéfini et la présence du quantifieur « many », par exemple. En effet, selon P. Martinez,

«Il peut y avoir interférence, c'est-à-dire emprunt momentané et involontaire d'une forme du systèmesource (anglophone: « sur le rue » pour « dans la rue », créolophone : « je suis parti» pour « je m'en vais ») ou création d'une forme mixte originale. » (33)

Le deuxième exercice proposé ressemble au premier et intègre le lexique étudié. Il n'y a pas de grande nouveauté grammaticale introduite à ce niveau, sauf l'article zéro devant les noms propres. Les règles de grammaire ont été répétées pendant la correction de l'évaluation. Les différents déterminants sont réactivés à l'occasion de l'élucidation des documents des séquences suivantes.

En ce qui concerne la troisième étape de cette expérimentation, l'absence d'article devant les noms d'activités a été étudiée, mais l'absence d'article devant « school» ( I go to Ø school») n'est pas justifiée ${ }^{5}$. Les substances et les notions abstraites ont souvent, en surface, le même comportement grammatical, donc la même détermination: $\varnothing+\mathrm{N}$ sing. Alors qu'ils correspondent à deux valeurs profondes différentes: /continu dense/ et /continu compact/. Pour les apprenants de L2, la dissymétrie «2 valeurs $\rightarrow 1$ forme » est assez incohérente. Mais ne dit-on pas «l'erreur est [d'ailleurs] souvent le signe qu'une règle est bien assimilée » (Philippe 44) ? Une erreur cache souvent des hypothèses sensées que l'élève formule sur le fonctionnement de la langue. Elle peut aussi révéler une application mécanique, ainsi qu'une absence de réflexion face à un problème. Seule une analyse approfondie des erreurs permet d'y voir clair. La plupart des méprises des apprenants sont considérées comme un problème de "calque » de L1 sur L2 ou d'interférence. Mais elles peuvent aussi porter sur des zones de résistance ou des domaines de vulnérabilité linguistique précis. La référence au générique ou à l'abstrait, au non-comptable, sont des exemples d'invariants du langage qui posent problème.

Voici le récapitulatif des trois exercices :

Exercice 1: complétez par l'article anglais qui convient: $a$ / an / Ø / ou the.

1.Matt is in ... library.

2.Mr Green is ... Irish teacher.

3.They are ... shoes.

4.She's ... friend.

5.Look at ... map!

Exercice 2: complétez par l'article anglais qui convient: $a$ / an / Ø / the.

1.They live in ... Chesil Street.

2.It's near ... river.

\footnotetext{
$\overline{5}$ Les apprenants français peuvent passer leur scolarité au collège à apprendre puis restituer « (to) go to $\varnothing$ school», «(to) be at Ø home », etc. sous forme de set phrases ou d'expressions à apprendre telles quelles et à reproduite sans faute, ce qui n'est pas toujours simple.
} 
3.Jakes lives in ... flat in Winchester.

4.It's next to ... leisure centre.

5.Look! There's ... owl on the tree!

Exercice 3: complétez par l'article anglais qui convient: $a$ / an / Ø / the.

1.Donovan is ... delivery man.

2.He works at ... Pizza Hut.

3.I like ... cats.

4.Holly is ... English girl.

5.Matt and Jake play ... football.

2. Instruments de mesure

La lecture des différents graphiques illustrant l'expérimentation va permettre de découvrir si les six groupes de Sixième pris au hasard ont un comportement identique confrontées au même procédé didactique. On va également pouvoir constater si la progression des résultats est régulière ou si elle suit un schéma " en $U$ », partant du principe que les performances des «bonnes classes»- suivant toute logique purement scolaire - seront supérieures à celles des classes plus faibles.

Figure 3 : résultats des six classes au troisième exercice (sur cinq points) sous forme de camemberts.
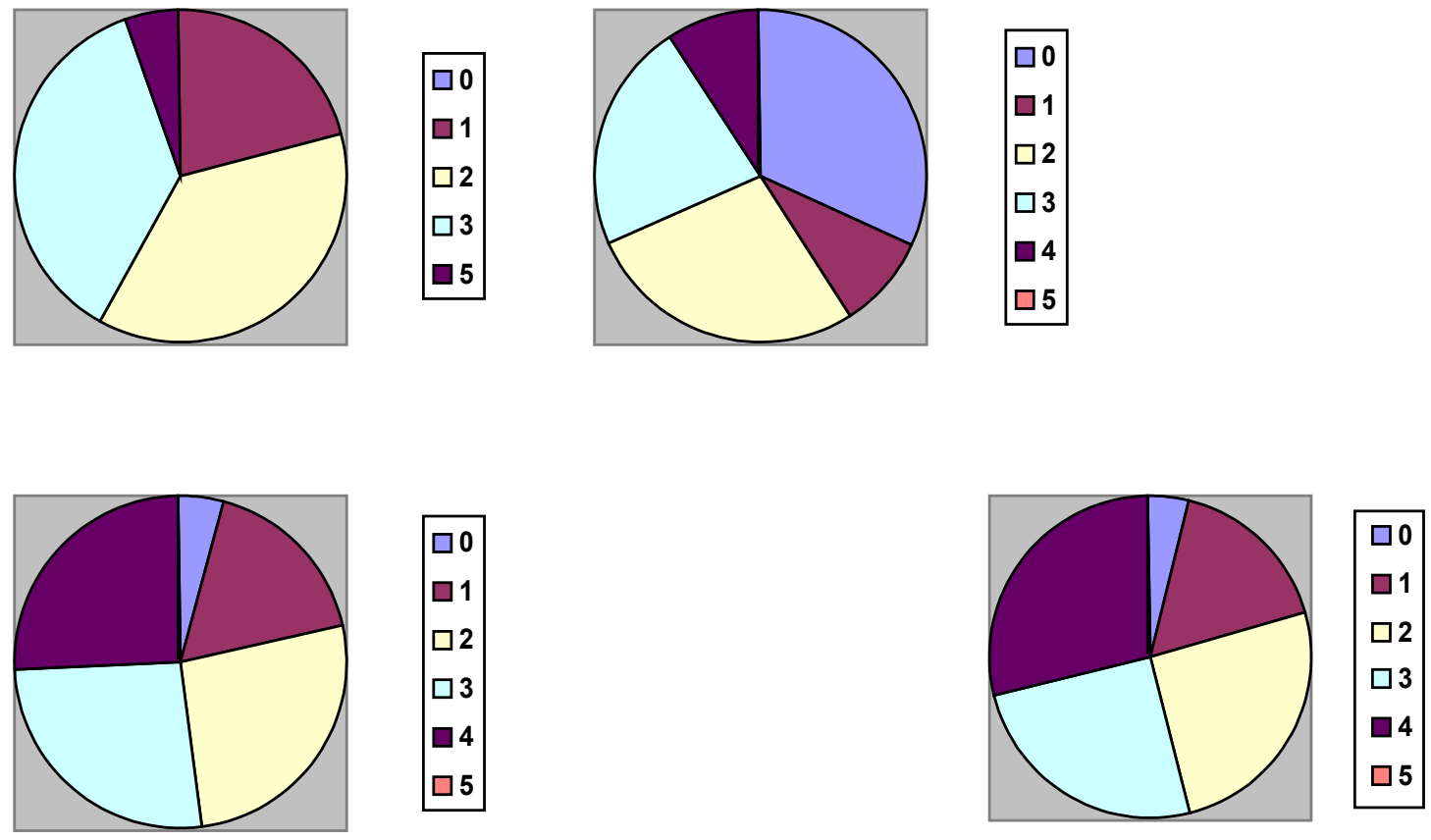

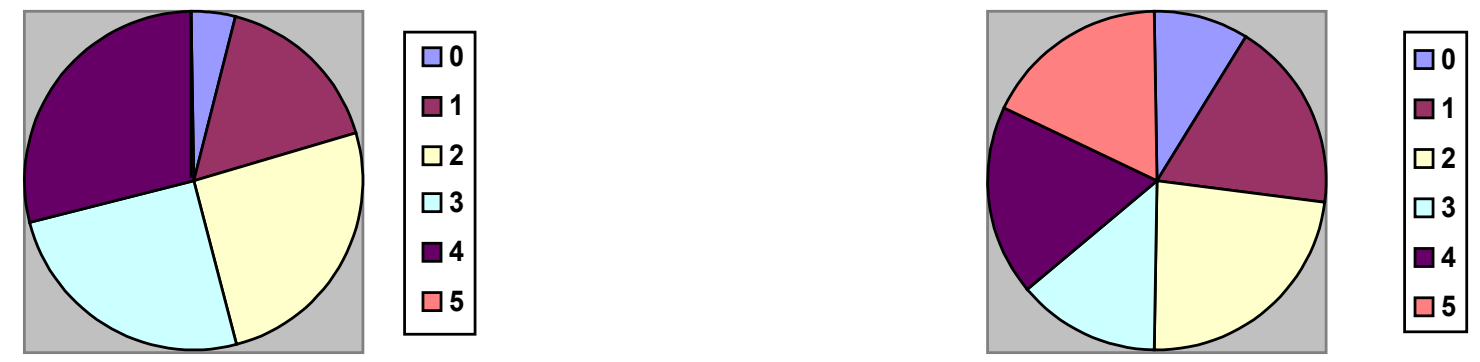

Chaque exercice était noté sur cinq points sans possibilité d'obtenir des demi-points. Ces résultats peuvent être représentés selon les deux graphiques suivants :

Figure 4 : représentations graphiques des moyennes des exercices donnés sous forme de tableau.
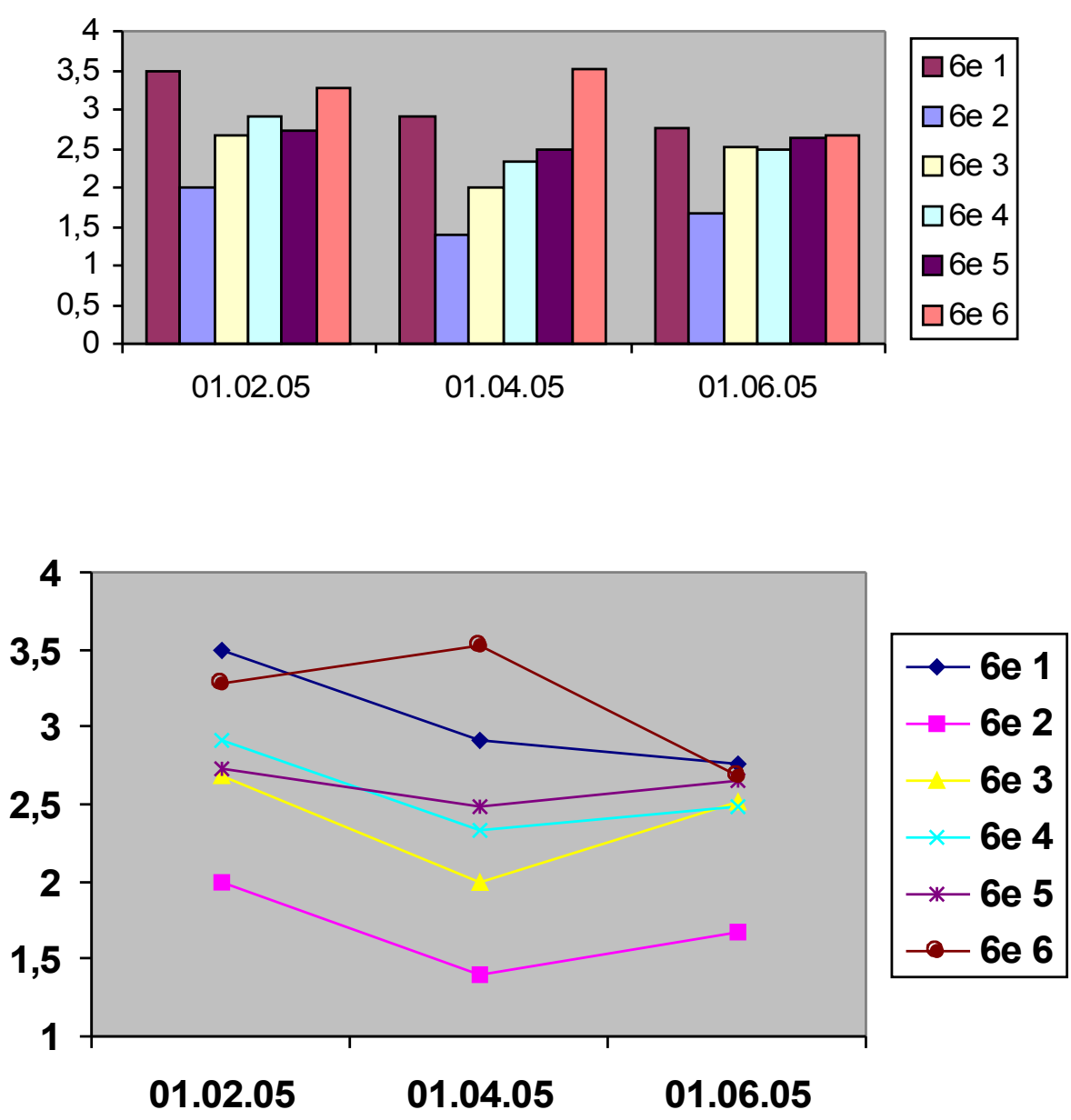

Les moyennes de chaque exercice par classe ont été réparties dans le tableau suivant:

Figure 5 : moyennes aux différents exercices proposés par date. 


\begin{tabular}{|c|c|c|c|}
\cline { 2 - 4 } \multicolumn{1}{c|}{} & Date $: 02 / 05$ & Date $: 04 / 05$ & Date $: 06 / 05$ \\
\hline Classe $: 6^{\mathrm{e}} 1$ & 3,5 & 2,92 & 2,76 \\
\hline Classe $: 6^{\mathrm{e}} 2$ & 2 & 1,4 & 1,68 \\
\hline Classe $: 6^{\mathrm{e}} 3$ & 2,68 & 2 & 2,52 \\
\hline Classe $: 6^{\mathrm{e}} 4$ & 2,91 & 2,33 & 2,48 \\
\hline Classe $: 6^{\mathrm{e}} 5$ & 2,73 & 2,48 & 2,65 \\
\hline Classe $: 6^{\mathrm{e}} 6$ & 3,28 & 3,52 & 2,68 \\
\hline Moyennes & 2,85 & 2,15 & 2,45 \\
\hline
\end{tabular}

\section{Résultats}

Quand on observe les moyennes aux exercices donnés, on constate qu'ils forment une courbe avec le côté gauche légèrement plus haut que le côté droit, et un "creux» qui peut être impressionnant pour une classe. Il est à remarquer cependant, que les résultats obtenus (sauf un) décrivent la même courbe, quelles que soient les options choisies par les élèves, et la composition des groupes (classes hétérogènes). Soignant leur performance trimestrielle d'anglais, nous pouvons penser que les apprenants ont révisé sérieusement leur grammaire pour avoir de bons résultats écrits au dernier test de la série (juin 2005), et corriger ainsi la note moyenne du deuxième exercice (avril 2005). Les options choisies par les apprenants doivent entrer en ligne de compte dans l'interprétation des résultats chiffrés, sachant qu'un des groupes concernés est une classe «bi-langue ». Les élèves étudient, en effet, deux langues secondes dès la Sixième, soit l'anglais (trois heures hebdomadaires) et l'espagnol (trois heures également), alors que les autres n'étudient que l'anglais à raison de quatre heures par semaine. Les élèves de ces classes sont recrutés à la demande de leurs parents et sur les conseils du maitre de CM2, en fonction des résultats et de leurs possibilités de travail, le «dernier mot» revenant, à juste titre, aux enseignants. On estime qu'ils ont des capacités linguistiques supérieures aux autres à cause dur surcroît de travail occasionné par l'apprentissage supplémentaire de l'espagnol. La classe bi-langue est devenue un "dispositif» à La Réunion, c'est-à-dire qu'elle est organisée et mise en place en fonction des moyens de l'établissement. Ce n'est pas un projet national et les effectifs sont contingentés.

Les moyennes décrivant un schéma en « $\mathrm{U}$ » ne sont pas uniquement - comme on pourrait le croire - le résultat d'une efficace « reprise en main » pédagogique des élèves, dans une relation de cause à effet: plus de discipline en classe signifierait une hausse certaine des performances, parce que apprendre relève d'une conduite volontaire, alors que moins de discipline provoquerait la baisse des moyennes. Cette courbe illustre l'évolution cognitive normale de l'ensemble des apprenants d'une même classe d'âge, soumis à des exercices d'apprentissage similaires. Comme la recherche l'a prouvé, les conceptions en place dans la tête de l'élève rejettent toute nouvelle information qui ne les conforte pas. L'apprentissage linguistique demande du temps et de la patience.

La discipline scolaire est un paramètre à prendre en compte dans l'ensemble des résultats, mais, ne nous en déplaise, ce n'est pas le facteur décisif en matière d'acquisition cognitive.

\section{Discussion}

Selon les moyennes obtenues pendant un semestre d'expérimentation sur six classes de Sixième, il semblerait que les performances des élèves concernés suivent une courbe d'apprentissage «normale » d'après les spécialistes, soit une courbe en «U», dans des conditions classique 
d'acquisition de la L2. Ce résultat est à moduler en fonction des connaissances antérieures de chacun des apprenants et de la composition hétérogène des groupes ${ }^{6}$. Il va de soi que la pédagogie de chaque enseignant concerné par ces groupes est un facteur non négligeable dans les résultats de ce type de recherche pédagogique, mais il est très difficile à évaluer de façon chiffrée et scientifique. Il existe un « effet enseignant » comme il existe un « effet établissement».

Cette étude révèle également l'écart important qui existe entre les connaissances apprises et les connaissances réelles des apprenants. Celles-ci dépendent avant tout de leur utilité. La grammaire anglaise passe auprès des apprenants pour difficile, voire opaque. Les règles d'usage de la pratique scolaire n'ont d'intérêt durable chez les jeunes. Il y a une tension permanente parmi les professeurs entre les savoirs prescrits par l'école et les savoirs construits par les élèves, ce qui fait affirmer à J-L. Chabanne :

« [...] il nous apparait que l'élève, qu'il soit placé « au centre » ou à la « périphérie » du système éducatif, reste toujours figuré par une distance mesurée entre la réalité personnelle, ce qu'il est dans sa personne, et un ailleurs où sa place serait idéale; il est représenté à la mesure du manque en tre l'apparence de ce qu'il est (ses compétences) et l'apparence de ce qu'il n'est pas (les compétences attendues). Il est une différence. » (16)

Ces résultats ont également permis de mettre en évidence le facteur « neutre » du créole chez ces jeunes apprenants en milieu scolaire francophone. Le fait qu'ils parlent cette langue en famille, voire à l'école ou au collège, dans un département français de l'Océan Indien, ne semble pas avoir de conséquence négative sur l'apprentissage de la L2. Les élèves passent d'une langue à l'autre avec difficulté parfois - mais réussissent à « cloisonner » leurs savoirs au même titre que ceux de Métropole. A ce niveau uniquement, nous pouvons affirmer que le créole n'est pas un obstacle à l'acquisition d'une langue étrangère seconde comme l'anglais. Aurait-elle alors un impact positif? Mon expérience personnelle d'enseignante dans le secondaire depuis huit ans à La Réunion tendrait à prouver le contraire en militant pour un impact relativement neutre sur l'appropriation des savoirs linguistiques autres que la langue maternelle.

\section{Conclusion}

La Réunion ne constitue pas un modèle de référence dans le domaine de l'enseignement des langues vivantes étrangères. Les résultats aux évaluations nationales des élèves de Sixième se sont révélés médiocres et les performances de beaucoup de lycéens décevantes malgré des années d'apprentissage. La création récente d'une Licence de didactique du français langue étrangère et seconde à l'université de Saint-Denis illustre une situation de plurilinguisme local complexe.

Les processus d'acquisition naturels de la langue première (LV1) et d'une langue seconde (LV2) comme l'anglais sont différents. Ces divergences, source de fragilité lors de l'initiation linguistique chez l'apprenant francophone, ont été analysées par le biais d'un cas précis: l'acquisition de la détermination nominale par des élèves de six classes de Sixième d'un collège du sud de La Réunion. Trois exercices de type lacunaire identiques ont été donnés à six groupes différents d'élèves d'une même classe d'âge sur une période d'un trimestre. Les moyennes ont été systématiquement relevées puis analysées au regard de spécialistes comme D. Bailly. Les résultats obtenus en 2004-2005 montrent que, malgré la composition des classes, les options choisies par les enfants ou leur "passé » linguistique, l'évolution cognitive en matière d'acquisition grammaticale suit toujours ou presque un schéma similaire. Cette courbe en $U$ prouve entre autres que la langue créole n'est pas un obstacle à l'assimilation de l'anglais langue seconde, mais a plutôt un rôle neutre dans ce cas précis. Des études intéressantes menées en Allemagne (voir

\footnotetext{
${ }^{6}$ La journaliste Gaëtane Chapelle rappelle que «Dans les classes hétérogènes (où l'on mélange les enfants de tous niveaux) [par contre], les élèves faibles y progressent beaucoup mieux avec un léger détriment pour les élèves forts. » (Ruano-Borbalan 133)
} 
Alternative Francophone vol.1, 3(2010), 128-140

http://ejournals.library.ualberta.ca/index.php/af

Hufeisen) axées sur l'exploitation des erreurs et interférences L1-L2 sont à suivre dans le futur. Leurs résultats pourraient conduire les didacticiens des langues à La Réunion à repenser et réorganiser la pédagogie linguistique à venir, voire même à utiliser la langue créole au collège au lieu de la stigmatiser. 


\section{Bibliographie}

Alamichel, Marie-Françoise. Pronoms et déterminants. Paris : Ellipses, 1999.

Bailly, Danielle. L'acquisition de la détermination nominale. Cahiers de Recherche numéro spécial. Gap : Ophrys, 1990.

Bailly, Danielle. Didactique de l'anglais. Vol. 1 : objectifs et contenus de l'enseignement. Paris : Nathan, 1997.

Bailly, Danielle. Didactique de l'anglais, vol. 2 : la mise en oeuvre pédagogique. Paris : Nathan, 1998.

Calvet, Louis-Jean. Linguistique et colonialisme. Paris : Petite Bibliothèque Payot, 1974 [2002].

Culioli, Antoine. La théorie d'Antoine Culioli. Ouvertures et incidences. Gap : Ophrys, 1992.

Culioli, Antoine. Pour une linguistique de l'énonciation. Tome 2. Gap : Ophrys, 1999.

Culioli, Antoine. Pour une linguistique de l'énonciation. Tome 3. Gap : Ophrys, 1999.

Culioli, Antoine. Pour une linguistique de l'énonciation. Tome 1. Gap : Ophrys, 1990 [1999].

Culioli, Antoine. Variations sur la linguistique. Paris : Klincksieck, 2002.

Dodge, Chantal et Martina, Claudine. L'anglais à l'école et au collège. Paris : Belin, 1989.

Dortier, Jean-François (coordonné par). Le langage. Nature, histoire et usages. Auxerre : Éditions Sciences Humaines, 2001.

Dubois, Jean et al. Dictionnaire de linguistique. Paris : Larousse, 2002.

Fournier, Martine et Lécuyer, Roger (coordonné par). L'intelligence de l'enfant. Le regard des psychologues. Auxerre : Éditions Sciences Humaines, 2006.

Groussier, Marie-Line et Rivière, Claude. Les mots de la linguistique. Lexique de linguistique énonciative. Gap : Ophrys, 1996.

Guillemin-Flescher, Janine. Syntaxe comparée du français et de l'anglais. Problèmes de traduction. Gap : Ophrys, 1993.

Lapaire, Jean-Rémi et Rotgé, Wilfrid. Réussir le commentaire grammatical de textes. Paris : Ellipses,1992. Martinez, Pierre. La didactique des langues étrangères. Paris : Presses Universitaires de France, 1996.

Morel, Fiona et al. New Wings 6e. Paris : Belin, 1998.

Morel, Fiona et al. New Wings 6e. Workbook. Paris : Belin, 1998.

Morel, Fiona et al. New Wings $6^{e}$. Livre du professeur. Paris : Belin, 1998.

Neveu, Franck. Lexique des notions linguistiques. Paris : Nathan Université, 2000.

Puren, Christian et al. Se former en didactique des langues. Paris : Ellipses, 1998.

Quivy, Mireille et Tardieu, Claire. Glossaire de didactique de l'anglais. Paris : Ellipses, 2002.

Souesme, Jean-Claude. Apprentissage grammatical de l'anglais. Collège-Lycée. Illustration d'une approche énonciative. Nice : CRDP académie de Nice, 2003.

Ruano-Borbalan, Jean-Claude (coordonné par). Éduquer et former. Les connaissances et les débats en éducation et en formation. Auxerre : Sciences Humaines Éditions, 2001.

Sournin Dufossé, Sophie. Les théories linguistiques, les pratiques pédagogiques et l'acquisition de la détermination nominale chez. les apprenants francophones, thèse de doctorat non publiée, Université de La Réunion, 2007.

\section{Articles consultés}

Altet, Marguerite. «Les styles pédagogiques ». Éduquer et former. Ruano-Borbalan Jean-Claude (dir.), Auxerre : Éditions Sciences Humaines, 2001 : 201-205.

Beniamino, Michel. «Le français réunionnais », www.bibliotheque.refer.org, 1996. Web. 12 Oct. 2010.

Chabanne, Jean-Luc. Actes du séminaire "Prévention contre l'illettrisme », Lancement de la formation des enseignants appelés à travailler dans les nouveaux dispositifs d'aide aux élèves en grande difficulté au collège, Académie de La Réunion, 2006 : 1-33.

Chapelle, Gaëtane. "L'école, la classe ou le maitre? », in Hadji c. "Les voies de l'évaluation », Éduquer et former. Ruano-Borbalan Jean-Claude (dir.), Auxerre : Éditions Sciences Humaines, 
$2001: 131-138$.

Dodille, Norbert. "Littérature et diglossie à La Réunion des années 1970 à nos jours ", www.dodille.fr, 2009. Web. 12 Oct. 2010.

Gaonac'h, Daniel. «L'enseignement précoce des langues », Le langage. Dortier Jean-François (dir.), Auxerre : Éditions Sciences Humaines, 2001 : 271-280.

Grangeat, Michel. "Lev s. Vygotsky», Éduquer et former. Ruano-Borbalan Jean-Claude (dir.), Auxerre : Éditions Sciences Humaines, 2001:103-108.

Hufeisen, Britta.(2005), « Parler plusieurs langues: c'est facile! » Cervean \& Psycho n 11 , septembreoctobre 2005 : 46-50.

Lassagne, François. «Créole. La naissance d'une langue » Sciences \& Vie n 227, 2004 : 78-85.

Philippe, Karine. «Les linguistiques de la parole », Sciences Humaines, n¹59, 2005 : 44-45. 